Edwin Kreuzer

\title{
Ein Ansatz für den Umgang mit Risiken und Unsicherheit in der Entscheidungspraxis
}

\section{Kommentar zum Beitrag „Entscheidung in Unsicherheit - eine tägliche Herausforderung und Lösungsstrategien im Bereich der Feuerwehr"von Klaus Maurer}

Technische Schadenslagen aber auch Naturereignisse wie Erdbeben, Überschwemmungen, Erdrutsche und Stürme bedrohen Menschen und verursachen weltweit immense volkswirtschaftliche Schäden. Auf internationaler Ebene wurden Vereinbarungen getroffen, um den sozialen, wirtschaftlichen und ökologischen Folgen dieser Ereignisse besser begegnen zu können.

Bei vielen komplexen Situationen sind aber die Risiken überhaupt nicht von vornherein klar, die für die Auswahl der richtigen Entscheidung bekannt sein müssten. Gerade für die Einsatzleiter und Führungskräfte der Feuerwehr, die Entscheidungen auf der Grundlage unvollständiger Informationen und unter extrem hohem Zeitdruck zu treffen haben, trifft dies fast immer zu.

Der International Risk Governance Council (IRGC) mit Sitz in Lausanne ist eine unabhängige, spendenfinanzierte Organisation, die es sich zur Aufgabe gemacht hat, das Verständnis und das Management von neuen, auch systemischen, Risiken zu verbessern.

Das vom IRGC entwickelte Modell des Risiko-Governance-Prozess [1], siehe Abb. 6.7, wird aufgeteilt in einerseits den Erfassungsbereich (die Erzeugung von Wissen) und andererseits den Managementbereich (Entscheidung und Umsetzung von Maßnahmen), die beide zusammenwirken müssen! Für eine ausführliche Erläuterung dieses integrativen Ansatzes wird auf die Publikation von Renn und Sellke [2] verwiesen. Im Folgenden seien nur einige Hinweise zum Risiko-Governance-Prozess aufgeführt.

Der Prozess wird in drei Hauptphasen eingeteilt:

- Vorabbewertung

- Risikobeurteilung

- Risikomanagement

Eine weitere Phase, die Tolerierbarkeit \& Akzeptanzbeurteilung, unterteilt in Risikobewertung und Risikocharakterisierung, ist zwischen die Phasen der Risikobeurteilung sowie des Risikomanagements gelegt. Dies bringt zum Ausdruck, dass diese Aufgabe je nach konkreter Fragestellung entweder von den Verantwortlichen der Vorabbewertung oder von den Risikomanagern bearbeitet werden kann. Die fünfte Phase ist die Risikokommunikation, die mit allen anderen Phasen direkt verbunden ist. 
Managementbereich: Entscheidung \& Umsetzung von Maßnahmen
Erfassungsbereich: Erzeugung von Wissen

Vorabbewertung

- Problemeingrenzung (Framing)

- Frühwarnsysteme

- Screening

- Festlegung wissenschaftlicher

Standards

\begin{tabular}{l}
\multicolumn{1}{c}{ Risikomanagement } \\
Umsetzung \\
· Durchführung der Maßnahmen \\
- Überwachung \& Kontrolle \\
- Feedback vom Risiko- \\
management \\
Entscheidungsfindung \\
- Identifizierung \& Generierung \\
von Optionen zur Risiko- \\
minimierung \\
- Bewertung von Optionen \\
- Evaluation \& Auswahl von \\
Optionen
\end{tabular}

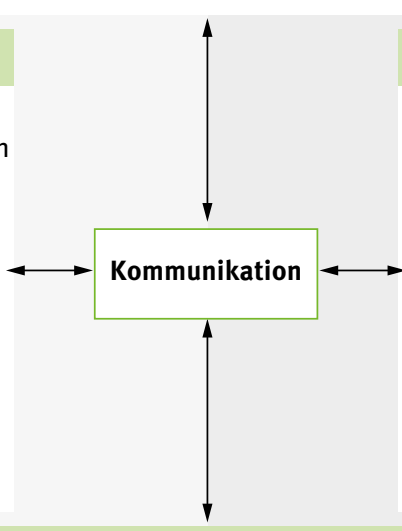

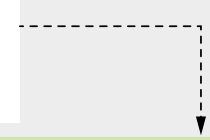

Risikobeurteilung

Risikoabschätzung

- Gefahrenermittlung \& -einschätzung

- Gefährdungspotential \& Exposition

- Risikoberechnung

Gesellschaftliche Risiko-

einstufung

- Risikowahrnehmungen

- Soziale Belange

- Sozioökonomische

Auswirkungen

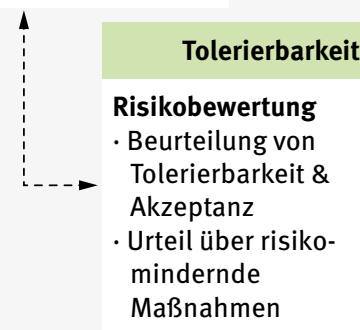

Akzeptanzbeurteilung

Risikobewertung

Beurteilung von

Tolerierbarkeit \&

Maßnahmen

\section{Risikocharakterisierung}

- Risikoprofil

- Beurteilung des

Gesamtrisikos

- Schlussfolgerungen \&

Optionen zur Risiko-

minimierung

Abb. 6.7 Der IRGC-Risiko-Governance-Prozess (siehe: http://irgc.org/wp-content/uploads/2012/ 04/IRGC_WP_No_1_Risk_Governance__reprinted_version_3.pdf).

Der Erfolg oder Misserfolg dieses Risiko-Governance-Modells ist natürlich vom vorhandenen Wissen über ein Risiko abhängig.

Deshalb ist es von entscheidender Bedeutung, das Wissen über ein Risiko systematisch zu kategorisieren. Dies ist ein weiterer zentraler Baustein des Ansatzes. Für den einfachsten Fall, bei dem die kausalen Zusammenhänge zwischen Ursache und Wirkung eines Risikos eindeutig und unbestritten gegeben sind, spricht man von einem simplen Risiko. In anderen Fällen, die durch ein kompliziertes Zusammenspiel mehrerer materieller und psychologischer Faktoren gekennzeichnet sind, spricht man von komplexen Risiken.

Soweit die Theorie! 
Der Mensch kann der Theorie nie gerecht werden. Extremsituationen und Unsicherheiten überfordern häufig unsere Fähigkeiten, und trotzdem müssen Entscheidungen getroffen werden. Hier ist es wichtig, dass in der Entscheidungspraxis der Feuerwehr (drillmäßig eingeübte) Automatismen greifen, die zudem aufgrund von Erfahrungen das Handeln mitbestimmen.

$\mathrm{Zu}$ beachten ist auch, dass unsere Rationalität in der Regel immer nur eingeschränkt zur Wirkung kommt. Deshalb wird in der Forschung heute von einer eingeschränkten Rationalität bei der Entscheidungsfindung ausgegangen, entgegen den vom Optimierungsgedanken geleiteten klassischen Annahmen. Eingeschränkt rationale Entscheidungen sind jedoch keineswegs immer irrational, wie aus anderen Beiträgen dieses Buches deutlich wird.

Eingeschränkte Rationalität basiert auf der Sichtweise, dass Entscheidungen im Rahmen der tatsächlichen kognitiven Möglichkeiten der handelnden Akteure getroffen werden. Entscheider müssen aber auch äußere Faktoren, wie Komplexität der Entscheidungsumwelt oder Zeitmangel, bei der Gestaltung einer Entscheidungsprozedur berücksichtigen. Die Entscheidungsprozeduren sind dabei durchaus vernünftig und führen gewöhnlich, aber nicht immer, zu guten Entscheidungen. Oder, wie der Autor des vorherigen Beitrags feststellt: „Die zweitbeste Entscheidung ist besser als keine Entscheidung“.

Aus den Ergebnissen der Forschung wurde für den praktischen Einsatz bei der Feuerwehr, dem Katastrophenschutz und der zivilen Verteidigung ein Regelkreis - eine kondensierte Form des integrativen Risiko-Governance-Prozesses - zur strukturierten Entscheidungsfindung entwickelt. Dieser Regelkreis wird im Laufe eines Einsatzes bei ständigem Zuwachs an Informationen und Adaption an neue Erkenntnisse fortwährend durchlaufen.

Für den Einsatz bei Gefahrenlagen in der Feuerwehrarbeit lassen sich die folgenden wesentlichen Elemente benennen:

\section{Die Herausforderungen}

- Eine oder mehrere Gefahren müssen unter hohem Zeitdruck gleichzeitig bekämpft werden

- Die Informationsbasis ist anfänglich meist lückenhaft

- Hohe Informations- und Kommunikationsdichte

- Arbeiten in ständig wechselnden, in der Regel unbekannten Umgebungen

- Hohes Maß an Eigengefährdung und emotionaler Belastung der Einsatzkräfte

\section{Die Lösung}

- Strukturierte Vorbereitung und Kenntnis der „Kalten Lage“ und der aktuellen „Eigenen Lage und Möglichkeiten“

- Reduzierung der Gefährdungsabschätzung auf neun (vgl. Tab. 6.1: Gefahrenmatrix zur Beurteilung der Lagen) mögliche Gefährdungen beschreibende Aspekte zur schnellen Erfassung der Risiken 
- Systematisierung der Abwägung zur Gefahrenabwehr und

- Kontinuierliche Weiterentwicklung der Erkundung, Bewertung und Befehlsgebung in einem Regelkreis

\section{Chancen}

- Aktive Informationsgewinnung

- Der Einsatzleiter als „Motor“ der Einsatzbewältigung

- Hohes Maß an Verantwortungsbereitschaft auf der Basis von Wissen und Erfahrung

- Es gibt immer mehrere Möglichkeiten der Einsatzbewältigung. Ziel ist es, die bestmögliche Lösung zu finden (Aspekte: Schnelligkeit, Ressourcenaufwand, Erfolgsaussicht, Nebenwirkungen, Kosten)

\section{Risiken}

- Unvollständige oder fehlerhafte Erfassung der Lage

- Fehlentscheidungen mit Gefahr für Leib und Leben gefährdeter Personen und eigener Kräfte sowie hohe materielle Verluste

- Zögerliches Entscheidungsverhalten oder Passivität führt zu höherem Risiko mit einem unstrukturierten Vorgehen der Einsatzkräfte und Dritter

- Höchstes Risiko: keine Entscheidung treffen

Im Modell des International Risk Governance Council werden die wissenschaftliche Erfassung, die gesellschaftliche und wirtschaftliche Dimension des Handelns miteinander verknüpft. Diese Verknüpfung wird auch in den Lösungsmechanismen der Feuerwehr, die zusätzlich das Zusammenwirken der an der Gefahrenabwehr beteiligten Organisationen zu koordinieren hat, deutlich. Nach einem Einsatz wird das Handeln der Akteure analysiert-danach werden gegebenenfalls Modifikationen der Entscheidungsstruktur abgeleitet - und bei Personenschäden wird es zu einer juristischen Überprüfung kommen, ob Entscheidungen der Einsatzleitung zur Schadensausweitung beigetragen haben oder nicht. Dabei wird es darauf ankommen, ob das technisch Mögliche mit dem gesellschaftlich Erwarteten angemessen verbunden wurde, um die Risiken für alle Beteiligten zu minimieren.

Der Beitrag des Leiters der Hamburger Berufsfeuerwehr liefert eine von großer Erfahrung getragene Darstellung der Umsetzung theoretischer Erkenntnisse und Konzepte aus der Entscheidungstheorie und zeigt auf, mit welchen Strategien Einsatzleiter und Führungskräfte der Feuerwehr trotz begrenzten Wissens, starker kognitiver Belastung und unter hohem Zeitdruck Entscheidungen treffen und ihr Handeln organisieren. 


\section{Literatur}

[1] Renn O. with an Annexes by Graham P. Risk Governance: Towards an Integrative Approach. Ed. by International Risk Governance Council (White Paper No. 1). Geneva: IRGC, 2005.

[2] Renn O, Sellke P. Risk-Governance: Ein neuer Ansatz zur Analyse und zum Management komplexer Risiken. In: Nova Acta Leopoldina NF. 2014;117(397):55-76. 
